\title{
Improving the Performance of HF Radio Networks in Presence of Interference: Frequency Hopping Automatic Link Establishment
}

\section{Afrooz Haghbin ( $\nabla$ a.haghbin@srbiau.ac.ir)}

Islamic Azad University Science and Research Branch https://orcid.org/0000-0003-1551-0348

Masoud Khodaverdizadeh

Islamic Azad University Science and Research Branch

Farbod Razzazi

Islamic Azad University Science and Research Branch

\section{Research Article}

Keywords: Automatic Link Establishment , Frequency Hopping , Jamming Scenarios , Throughput

Posted Date: February 21st, 2022

DOl: https://doi.org/10.21203/rs.3.rs-834512/v1

License: (c) (i) This work is licensed under a Creative Commons Attribution 4.0 International License.

Read Full License 
Noname manuscript No.

(will be inserted by the editor)

\title{
Improving the Performance of HF Radio Networks in Presence of Interference: Frequency Hopping
} Automatic Link Establishment

\section{Masoud Khodaverdizadeh}

Afrooz Haghbin

\section{Farbod Razzazi}

Received: date / Accepted: date

\begin{abstract}
Automatic Link Establishment (ALE) is crucial in channel assignment and data exchange in HF radio networks. The time of link establishment and its quality are two main concerns in this criterion establishment. In jamming environments, the quality of the channels is seriously influenced by the jammer signals. Frequency Hopping (FH) approaches supports communication links to overcome the jamming impairments, and the traditional ALE algorithm can be made more stable by using this approach. This paper has developed ALE algorithms to automatically establish links to handle different jamming scenarios and establish robust links to exchange data with the proposed FH-ALE system. Besides, in the proposed algorithm, we have considered the time of link establishment to be almost identical to the current

Masoud Khodaverdizadeh

E-mail: Masoud.Khodaverdizadeh@srbiau.ac.ir

Afrooz Haghbin

E-mail: A.Haghbin@srbiau.ac.ir

Farbod Razzazi

E-mail: F.Razzazi@srbiau.ac.ir

Department of Electrical and Computer Engineering, Science and Research Branch, Islamic Azad University, Tehran, Iran
\end{abstract}


ALE scenario. Hence, in the normal channel condition, the throughput value is approximately identical in proposed and traditional cases. The simulation results represent the superiority of the proposed FH-ALE approach in jamming environments.

Keywords Automatic Link Establishment · Frequency Hopping · Jamming Scenarios - Throughput

\section{Declarations}

\section{Funding :}

- This article is the result of a student activity and is not supported by any organization.

\section{Conflicts of interest/Competing interests :}

- Not applicable.

\section{Availability of data and material :}

- Not applicable.

\section{Code availability :}

- All simulation codes are available.

\section{Authors' contributions :}

- Because the automatic link establishment algorithms presented in the current articles and standards do not work well in the presence of intentional and unintentional interferences, In this paper, a new automatic link establishment algorithm based on frequency hopping methods is presented, which increases the efficiency of the system in weak channel conditions.

- Usually, in frequency hopping based systems, the throughput decreases due to the increase of link establishment time. In the proposed approach, the automatic link establishment time does not increase compared to the traditional mode, and in normal channel conditions, it can be reduced this time. 
- Mathematical model of the proposed method is assumed to be a two-way module using the markov chain. The appropriate method is selected to increase the throughput with this model.

\section{Ethics approval :}

- Not applicable.

\section{Consent to participate :}

- Not applicable.

\section{Consent for publication :}

- Not applicable.

\section{Introduction}

In tactical communications, frequency hopping $(\mathrm{FH})$ capability is exploited for robust communication links against intentional and unintentional interferences. Moreover, Automatic Link Establishment (ALE) is employed to set up an excellent quality link in the High Frequency (HF) regime and provide the networking ability efficiently and quickly. Hence, by combining these two techniques, one can establish an ALE network in an HF channel that is highly robust in data traffic and signaling control.

So far, the second, third, and fourth generations of ALE have been introduced. Second generation ALE (2G-ALE) is demonstrated in the MIL-STD188-141A [1], MIL-STD-188-141B [2]. In this generation, the general reference time is not present. All the idle nodes search all the frequencies in the preset list asynchronously to detect the transmission request. The transmission period is based on the complete search cycle of the receivers. The destination node will answer the request whenever it received the scanning call. Then, 
the data link is established so two nodes can communicate using data protocols such as 2G-ALE. The crucial drawbacks of the 2G ALE are its timeconsuming process, and nonrobustness of the link establishment [3]. Accordingly, third-generation ALE (3G-ALE) was represented in MIL-STD-188-141C and STANAG4538 [4],[5] to cover these drawbacks. In 3G-ALE, all the nodes in the network have a general reference time. Hence, the preset frequencies are searched synchronously. Since all destinations listen to the preset frequencies simultaneously, the receiver would not wait for a complete search cycle. Moreover, in 3G-ALE, the link establishment is designed for the poor channel scenarios [6]. Furthermore, the fourth generation ALE (4G-ALE) is introduced in MIL-STD-188-141D, [7] in which the data rate is increased, and the link is established rapidly. All these approaches are for the fixed frequency case, and they will be attacked by the preset frequency list jamming.

Due to the vulnerability of different generations of ALE to the jamming, we can transmit the protocol data units (PDUs) in ALE by the FH technique to prevent interference. If the previously announced standards of HF are utilized, fixed frequency channels are substituted by the frequency sets in ALE. We can meet the goal by two different approaches in ALE. In the first approach, the link is established between all the network resources using a fixed frequency channel, and then data is transmitted by the $\mathrm{FH}$ links [2]. This approach is called Link Before Hopping (LBH). In the second approach, the establishment of the data links is based on the FH technique, and then the data are transmitted using the HF links [2]. This is called Link While Hopping (LWH).

Frequency hopping methods has traditionally been used to establish jammingresistant communication. Various standards and articles have used this method, Including STANAG 4444 [8] which is the NATO technical standard for slow hop HF electronic protection measures(EPM) communication system. In [8], a communication system with frequency hopping capability in the HF channel is expressed. The procedure involves two distinct waveforms called Enhanced Equalizer Waveform (EEWF) and Direct Sequence Waveform (DSWF). EEWF 
provides more data rates than DSWF in appropriate channel status, while the DSWF is utilized to establish robust communication in uneven channel status by frequency hopping communication links [8]. There are various approaches for the receiving process of FH signals. Concisely, in [9] three different criteria are demonstrated based on the hard decision, soft decision, and RAKE detection. The link establishment in [8] is a full-duplex and is compromised beforehand between transmitter and receiver. While in the proposed method in our work, the communication link is established automatically.

Regarding usual FH systems expressed in [8], the frequency set is constant in various channel conditions. Due to the high probability of interfering in the communication, it is possible to disconnect in the event of interference. Adaptive frequency hopping $(\mathrm{AFH})$ systems are introduced where communication techniques are utilized to improve frequency hopping systems' performance. For instance, the receiver sends the channel estimation data to the transmitter, and the transmitter changes the hop set according to the current channel condition. In [10], AFH is considered as the communications technique in the point-to-point wireless communication approach. Although channel conditions are estimated in AFH-based systems, communication between transmitter and receiver is not automated. However, the proposed method in this article is based on automated transmission. Also, recently, machine learning and cognitive approaches are exploited in FH to select the optimal channel set, which yields performance improvement encountering intentional interferences $[11],[12],[13]$.

This paper has demonstrated a Frequency Hopping Automatic Link Establishment (FH-ALE) approach to overcome the drawbacks of traditional ALE algorithm. While the timing is not increased significantly and in poor channel conditions, it is possible to establish the link automatically. Furthermore, the proposed criterion is robust in poor channel scenarios. There are three main contributions which are listed as follows: 
- Because the automatic link establishment algorithms presented in the current articles and standards do not work well in the presence of intentional and unintentional interferences, In this paper, a new automatic link establishment algorithm based on frequency hopping methods is presented, which increases the efficiency of the system in weak channel conditions.

- Usually, in FH-based systems, the throughput decreases due to the increase of link establishment time. In the proposed approach, the automatic link establishment time does not increase compared to the traditional mode, and in normal channel conditions, it can be reduced this time.

- Mathematical model of the proposed method is assumed to be a two-way module using the markov chain. The appropriate method is selected to increase the throughput with this model.

The remainder of the paper is organized as follows: In section 2, the System model of the proposed FH-ALE system is explained. Then the Mathematical Model for the FH-ALE Protocol is demonstrated in section 3. The simulation results and concluding remarks are represented in sections 4 and 5, respectively.

\section{System Model of Proposed FH-ALE Protocol}

In the traditional ALE, a list of frequencies is selected as a sweep list. The transceiver will assess each channel quality by transmitting and receiving Burst Waveform(BW) [5]. At last, one of the channels will be selected for communication according to the origin's request. In the proposed FH-ALE system, instead of a fixed frequencies list, there is a hop sets list that finally a suitable hop set is selected. Optimal hop set selection is based on the stored data in the LQA table. This data, which are the same values like the quality of the frequency channels, can be determined in the sounding phase by measuring the sent packets' quality or measuring the quality of the received PDUs during link establishment or data traffic. Sounding is the regular broadcasting of the specific signal sent from one node to another, making it possible to assess 


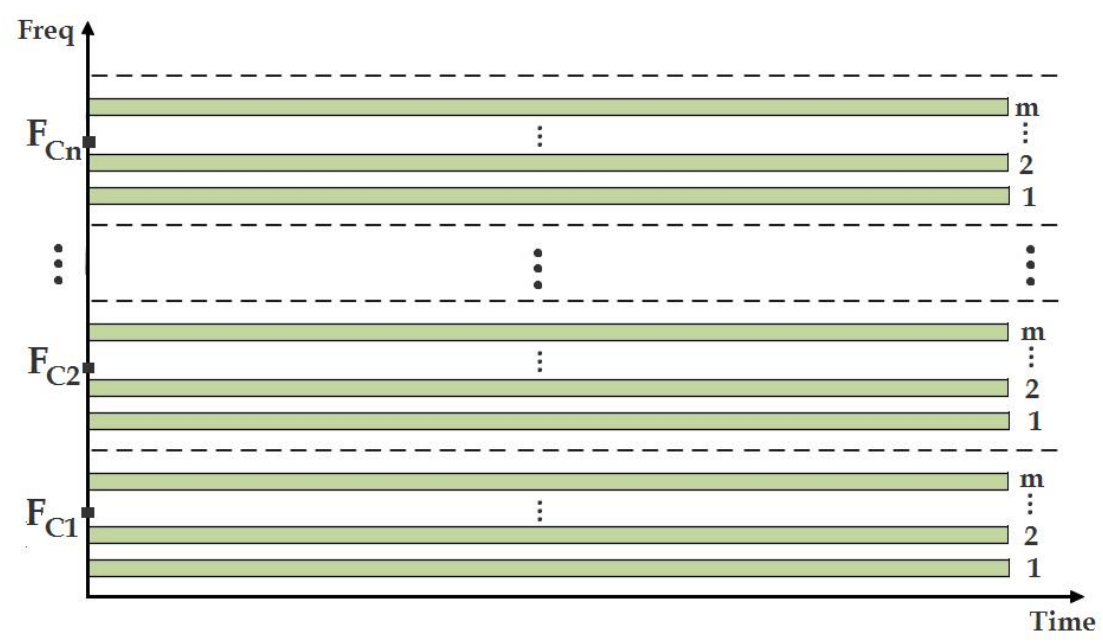

Fig. 1 Dividing channels in proposed FH-ALE system

the quality of the communication between these nodes. Details of sending the sounding signal and the optimal time intervals required to send it to update the LQA table values are given in MIL-STD-187-721D [14]. In the sounding phase, the recalling node transmits the hopping pattern in each sounding interval for the LQA table filling, using received SNRs in the receiver nodes. However, the LQA table can be initialized according to environmental conditions and geographical location between different nodes, using tools such as AREPS or VOACAP, and its details are discussed in [15],[16],[17],[18].

After filling the LQA table, the best channels with suitable conditions for transmitting data are selected, and the frequency hopping link is established. For channels quality assessment, the system bandwidth is divided into $n$ hopping intervals. As illustrated in Fig. 1, in each frequency interval, $m$ hopping channels are assumed. Selected channels can be distributed equally spaced in the frequency intervals and identically in all the communication nodes.

A particular pattern in each frequency interval is utilized in the rest of the paper, dedicated to the transmitter and the receiver before the transmission. For example, the determined pattern, in the $k^{t h}$ interval with central frequency $F_{c k}$, is demonstrated by black boxes in Fig. 2. The channels set $C_{k}$ is defined 


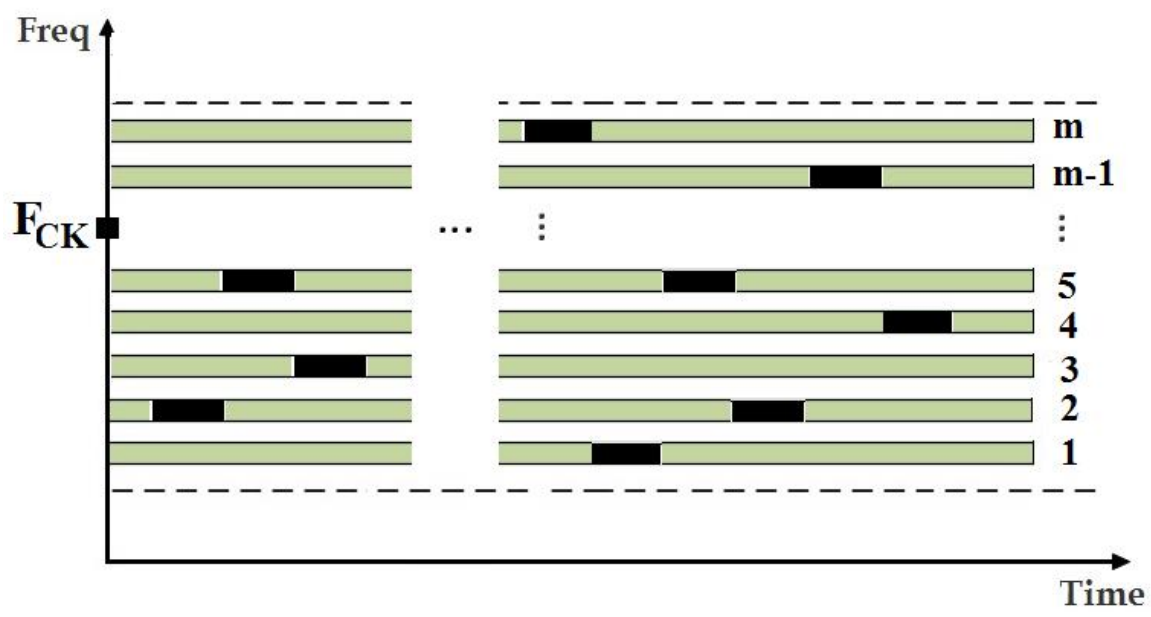

Fig. 2 A sample pattern for transmitting cluster waveform in the $k^{\text {th }}$ frequency interval

as

$$
C_{k}=\left\{c_{k 1}, c_{k 2}, \ldots, c_{k m}\right\}
$$

The assumed pattern includes all the frequencies in the set $C_{K}$, which is defined by a pseudo-random sequence generated using a Linear Feedback Shift Register (LFSR). The initial condition of the LFSRs in all the nodes are identical for synchronous order generation [19]. These patterns are determined in each frequency interval for link establishment and evaluation of the quality of the communication. The initial condition of the LFSR could be different in each interval.

In the traditional generations of ALE, a score is dedicated to each channel, and then the channel with maximum point is selected for link establishment [4],[20]. In the proposed frequency hopping scenario, a pattern with a maximum point can be selected. So, first, the channels with unacceptable conditions are omitted from the hopping list. Consequently, the new set is generated as

$$
C_{k}^{\prime}=\left\{c_{k 1}^{\prime}, c_{k 2}^{\prime}, \ldots, c_{k q}^{\prime}\right\}
$$

where $q \leq m$, and $C_{k}^{\prime}$ is the set of remained appropriate channel in the $k^{t h}$ interval. All the $n$ intervals are evaluated, and their appropriate channels are 
determined. Finally, using the LQA table results, the frequency interval with the maximum number of appropriate channels is selected for communications. Then, a unique pattern is compromised between the transmitter and the receiver for data transmission. This pattern includes the channels with satisfactory conditions in which an LFSR dictates the channel frequency sequence.

According to the asynchronous fixed frequency ALE model defined in [5], the traditional ALE process can be described by five states, channel listening, scanning call, response, acknowledgment, and data transmission. These five states provide a three-way automatic link establishment[21]. After selecting the appropriate frequency channel, the transmitter sends the scanning call signal sequentially equal to the scan list frequencies. The receiver is sweeping this list of frequencies to detect the transmitted scanning call signal. Then, the transmitter waits for a response from the receiver, and if it receives a response signal, it sends the acknowledgment signal, and finally, the link is established. A suitable frequency interval is selected to show the FH link in the asynchronous FH-ALE system instead of sending BW consecutively at a fixed frequency channel. The scanning call signal is sent sequentially with a specific pattern. The receiver also sweeps the various intervals to detect the scanning call signal and responds to the transmitter to establish the hopping link. The flowchart of the automatic link establishment operation in the frequency hopping mode is shown in Fig. 3.

As a result of the proposed system, two-way handshaking will be done for the link establishment. First, the transmitter listens before transmit (LBT) and assesses the probability of occupancy of each channel. Subsequently, the scanning call (SC) signal will be transmitted to the receiver. Then, the transmitter waits for the receiver's answer in Listen for Response (LFR) time interval. The receiver will investigate the occupancy status of the channels and notify the transmitter. If all the channels' occupancy status is appropriate in the two-way handshaking process, the ALE control signal will be exchanged, and the hopping link will be established. This process is depicted in Fig. 4. In the occupancy determination stage, either in the transmitter or in the receiver, 

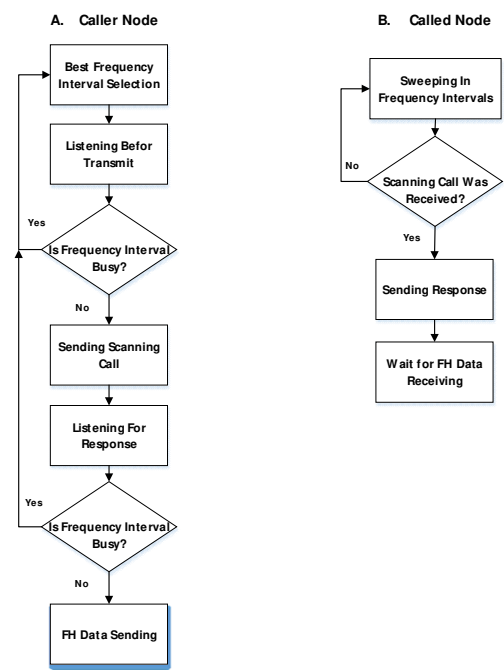

Fig. 3 Traffic establishment in the asynchronous proposed FH-ALE system, A- caller node, B- called node

the subsequent channels will be processed in each channel is occupied. Some limitations will be discussed in the following sections to select an interval.

The probability of $i^{t h}$ frequency occupancy in the transmitter is denoted by $\delta_{T X}^{i}$, which shows the probability that received signal strength indication (RSSI) in the corresponding channel is more than the predefined threshold. i.e.,

$$
\delta_{T X}^{i}=P\left(R S S I_{T X}^{i}>R S S I_{T h}\right)
$$

where $R S S I_{T h}$ represents the threshold RSSI for determining the occupancy of the channel and $R S S I_{T X}^{i}$ is the received RSSI in the $i^{t h}$ channel. If the channel conditions on the transmitter node are suitable for link establishment, these conditions are also checked on the receiver node. In the receiver, the occupancy probability is determined by comparing the corresponding channel SNR to a threshold value. Moreover, the occupancy probability can be formulated as

$$
\delta_{R X}^{i}=P\left(S N R_{R X}^{i}<S N R_{T h}\right)
$$




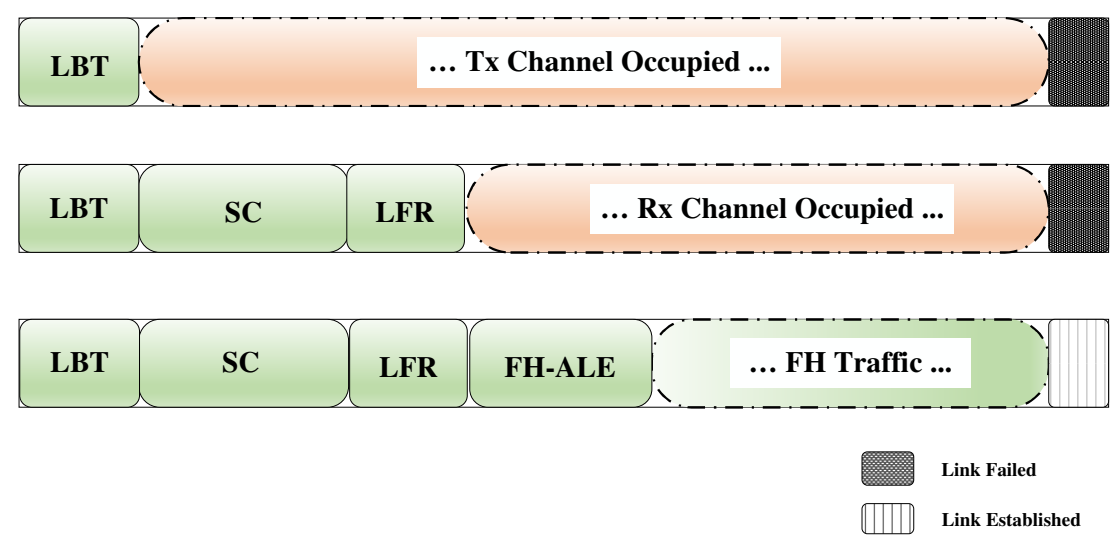

Fig. 4 TX and RX condition assessment for proposed FH automatic link establishment

where $\delta_{R X}^{i}$ and $S N R_{R X}^{i}$ denote the occupancy probability and the receiving $i^{t h}$ channel SNR respectively. $S N R_{T h}$ is the threshold SNR value. Hence, if the selected interval is not occupied both in transmitter and receiver, the data link will be established.

\section{Mathematical Model for the Proposed FH-ALE Protocol}

According to the proposed FH-ALE protocol, the considered frequency interval is divided into $n$ hopping interval. Each is partitioned into a $m$ hopping channel. It is assumed that $i$ is the appropriate interval index for establishing the frequency hopping link and is obtained using the results of the LQA table. In the listening period, the occupancy condition of $m$ channels in the $i^{t h}$ interval is evaluated. The channel occupancy probability is measured by $\delta_{T X}^{i, k}$ where $i, k$ represents the $k^{t h}$ channel in the $i^{t h}$ interval. Subsequently, the 


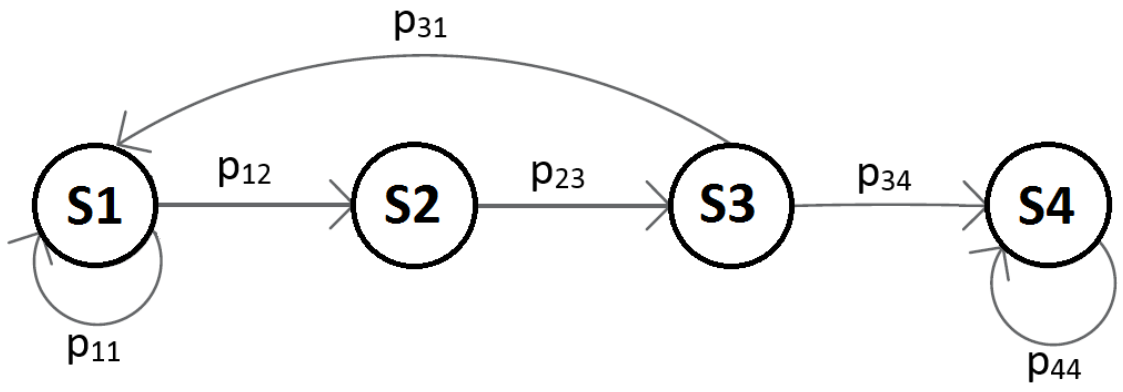

Fig. 5 Discrete time markov chain of the proposed FH automatic link establishment

received channel status will be evaluated and denoted by $\delta_{R X}^{i, k}$ in the receiver side by scanning call transmission. We have modeled the link establishment process by a Markov chain shown in Fig. 8. It is shown that the throughput of an ALE-based system is increased by decreasing the time of link establishment [21]. Consequently, the proposed approach in this paper is evaluated by time consumption in link establishment. According to the proposed link establishment, a two-way handshake is utilized for solidification, which involves the following four states of the Markov chain.

In state $S 1$, which is called listen before transmit (LBT), the recalling node selects the $i^{\text {th }}$ interval according to the LQA table. Here, $m$ channels are evaluated to determine the occupancy status in listening time $\left(T_{L B T}\right)$. The selected interval will be omitted from the proper channel list if at least $d$ channels from $m$ channels are detected to be occupied. Hence, having lower than $d$ occupied channels will activate the second state called Scanning Call (SC). If we denote the probability of transition from $S 1$ to $S 2$ as $p_{12}=1-P_{T X}$, then $P_{T X}$ can be modeled as

$$
P_{T X}=\sum_{k=1}^{d-1}\left(\left(\begin{array}{c}
m \\
k
\end{array}\right)\left(\prod_{j \in J} \delta_{T X}^{j}\right)\left(\prod_{j \in \bar{J}}\left(1-\delta_{T X}^{j}\right)\right)\right)
$$

where the $\delta_{T X}^{j}$ is the occupancy probability for $j^{\text {th }}$ channel in the transmitting side. $J$ is a subset of $J_{m}=1,2, \ldots, m$ and $\bar{J}$ is its complement. The occupancy probability of the selected interval is $p_{11}=P_{T X}$. In $S 2$, the SC has 
transmitted to the receiver, then the current state changes to the $S 3$. So $p_{23}$ is equal to 1 . In $S 3$, which is called Listen for Response (LFR), the occupancy status of the channel is evaluated. As mentioned above, if at least $d$ channels of $m$ channels have lower SNRs than the referenced $\operatorname{SNR}\left(S N R_{T h}\right)$, the selected interval is disqualified in the receiver, then the current state changes to $S 1$ to evaluate next interval. Otherwise, the current state changes to state $S 4$. Let $p_{34}=1-P_{R X}$ denote the probability of transition from $S 3$ to $S 4$, then $p_{31}=P_{R X}$ can be modeled as

$$
P_{R X}=\sum_{k=1}^{d-1}\left(\left(\begin{array}{c}
m \\
k
\end{array}\right)\left(\prod_{j \in J} \delta_{R X}^{j}\right)\left(\prod_{j \in \bar{J}}\left(1-\delta_{R X}^{j}\right)\right)\right)
$$

Ultimately, the system transits to the last state called data transmission denoted by $S 4$ and remains in this state until the end of data transmission; in other words, $p_{44}=1$. There are two cases which can cause the link failure. The first case is that the system in state $S 1$ has the failure time $T_{F 1}$, which is formulated as

$$
T_{F 1}=T_{L B T}=\sum_{k=0}^{m} T_{L B T}^{k}
$$

where $T_{L B T}^{k}$ is the listening time of $k^{t h}$ channel in the selected interval. The probability of link failure is

$$
P_{F 1}=p_{11}=P_{T X}
$$

The second case is that the system in state $S 3$ has the failure time $T_{F 2}$, can be modeled as

$$
T_{F 2}=T_{L B T}+T_{S C}+T_{L F R}
$$

where $T_{S C}$ is the required time to send $S C$, and waiting time $\left(T_{L F R}\right)$ is the required time for LFR. The probability of link failure in the second case is calculated as

$$
P_{F 2}=p_{12} p_{23} p_{31}=\left(1-P_{T X}\right) P_{R X}
$$

If the hopping frequencies between transmitter and receiver are not occupied, the link will be established. The probability of successful link establishment is 
represented as

$$
P_{S}=p_{12} p_{12} p_{34}=\left(1-P_{T X}\right)\left(1-P_{R X}\right)
$$

Consequently, the time of link establishment is denoted by

$$
T_{S}^{l}=T_{L B T}+T_{S C}+T_{L F R}+T_{A L E}
$$

where $T_{A L E}$ is the required time for transmitting various BWs. If in the $i^{\text {th }}$ interval the link is established while the previous $i-1$ intervals where unsuccessful, the establishment time is denoted by Eq. 13 where $T_{s}^{i}$ is the average time of link establishment.

$$
\begin{aligned}
T_{s}^{i}= & \sum_{k=1}^{i-1}\left(\begin{array}{c}
i-1 \\
k
\end{array}\right)\left(P_{F 1}\right)^{k}\left(P_{F 2}\right)^{i-1-k} \\
& \left(k T_{F 1}+(i-1-k) T_{F 2}+T_{s}^{l}\right) \\
= & \sum_{k=1}^{i-1}\left(\begin{array}{c}
i-1 \\
k
\end{array}\right)\left(P_{T X}\right)^{k}\left(1-P_{T X} P_{R X}\right)^{(i-1-k)}\left(k T_{L B T}+\right. \\
& \left.(i-1-k)\left(T_{L B T}+T_{S C}+T_{L F R}\right)+T_{\text {suc }}\right)
\end{aligned}
$$

We know that by reducing the time of link establishment, the throughput is increased[21], and according to the Eq.(13), reducing the link establishment time depends on reducing the $T_{L B T}$ and $T_{L F R}$. Considering Eq.(7), total listening time $\left(T_{L B T}\right)$ is equal to the summation of $m$ channels listening time, and so is $m$ times greater than that of the fixed frequency ALE system's listening time. In 4G-ALE, a method similar to the suggested method in this work is used, which is called 'Staring.' Staring in [24] is introduced as a new approach for the link establishment in wideband systems in which all the available frequencies are identically processed. This approach will ensure instant link establishment [25],[26].

To reduce link establishment time, we have proposed to utilize a channelizer to evaluate all the channels simultaneously. Thus, the listening time to all the channels will be equal to the listening time of one channel in a fixed frequency case. The proposed channelizer is based on the methods used in $[22],[23]$. Besides, after listening, calling, and recalling states, the channelizer 
decreases their corresponding time related to outage time. Using existing processors, it is possible to hardware implementation of intended channelizer, and various articles, including [27],[28], have addressed this issue.

\section{Simulation and Numerical Results}

In this section, the simulation results are demonstrated to assess the performance of the proposed approach. The simulation is performed in traditional ALE[6],[21] and our proposed FH-ALE case. The payload bits are convolutionally encoded, block interleaved, and then mapped using Walsh sequences. The Walsh coded bits are modulated by 8-Phase Shift Keying (8-PSK) and hopped according to the selected hop set. The simulation parameters are listed in Table 1. According to [2] minimum required SNRs guaranteeing 25\%, 50\%, and $95 \%$ of link success probability $-10,-9$, and $-7 \mathrm{~dB}$, respectively for fixed frequency (Appendix $\mathrm{C}$ ) and are $-7,-6$, and $-4 \mathrm{~dB}$, respectively for frequency hopping systems(Appendix F). Finally, 1000 number of 26-bit packets are transmitted in different SNR values. The performance metric is the linking probability that according to the Packet Error Rate (PER) as follows:

$$
P_{\text {link }}=(1-P E R)^{2}
$$

The time of link establishment in both traditional fixed frequency ALE and proposed frequency hopping ALE systems are represented in Table 2. As demonstrated, the consuming time is almost equal for both systems. Hence, the throughput is practically identical in both systems. The results of the table 2 are extracted from averaging on 1000 runs in MATLAB software without any jamming and interference in a PC with Core i7-4500U CPU @ $1.80 \mathrm{GHz} 2.40$ $\mathrm{GHz}$ processor and 4.00GB RAM.

4.1 The performance comparison in normal channel condition

The performance of the proposed approach is compared to that of the traditional link establishment via simulations. As mentioned above, the probability 
Table 1 Simulation Parameters

\begin{tabular}{|c|c|}
\hline Parameters & Values \\
\hline \hline Payload length & $26 \mathrm{bits}$ \\
Convolutional coding rate & $1 / 2$ \\
Interleaver & $4 \times 13$ block \\
Walsh sequence length & 64 \\
Modulation & $8-\mathrm{PSK}$ \\
Carrier frequency & $1.8 \mathrm{KHz}$ \\
Symbol rate & $2.4 \mathrm{Ksps}$ \\
Pulse shaping delay & 5 \\
Roll-off factor & 0.2 \\
Number of channels & 128 \\
Hopping set length & 64 \\
\hline
\end{tabular}

Table 2 Time consumption in the receiver of traditional ALE and proposed FH-ALE systems

\begin{tabular}{|c|c|}
\hline Time of traditional ALE system[6] & Time of proposed FH-ALE system \\
\hline \hline $1.27(\mathrm{Sec})$ & $1.36(\mathrm{Sec})$ \\
\hline
\end{tabular}

of link establishment is denoted as the main performance metric in different SNRs. As depicted in Fig. 6, the performance of both approaches is approximately the same. It represents that the link establishment probability is not influenced by the hopping essence of the proposed system as expected because, according to the results shown in Fig. 6, the probability of link establishment in different SNRs is less than $0.05 \mathrm{~dB}$. In the following part, the impact of jamming is deduced on the performance of the proposed approach.

4.2 The performance comparison in different jamming scenarios

In this part, different jamming scenarios are considered to evaluate the performance of the proposed approach [29]. The jamming scenarios are partial band jammer, follower jammer, and smart multi-tone jammer. Partial band jammer interferes on the 16 middle hops out of 128 hops. A follower jammer is applied to the previously transmitted signal frequency. Smart multi-tone jammer is 


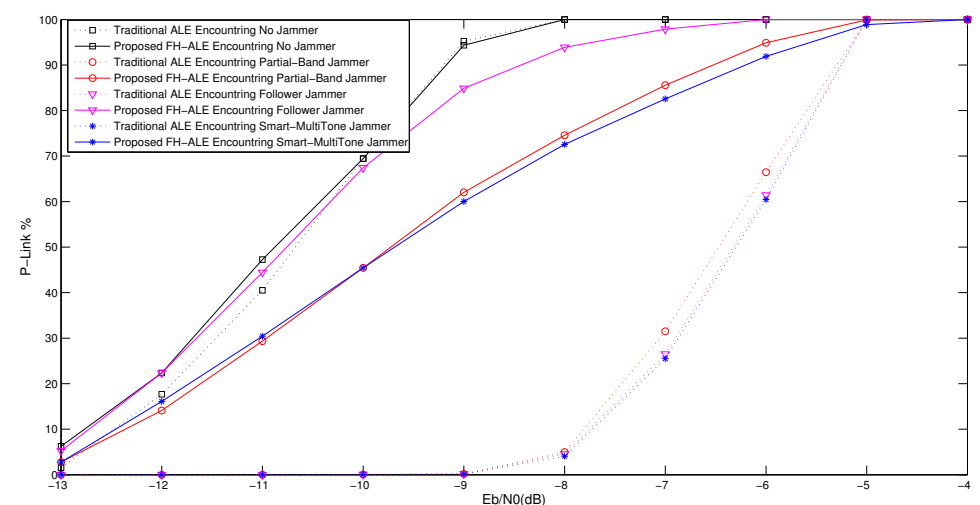

Fig. 6 The performance comparison of the proposed FH-ALE approach and the traditional ALE system[6] encountering various jamming environment

aware of the hopping set and interferes 16 hops in the middle of known hop set. Besides, in the fixed frequency case in the presence of jammer, one of the frequencies in the hop set is selected randomly for transmission.

Also, in Fig. 6 the proposed FH-ALE system performance is compared to that of the traditional ALE system in the presence of the partial band, follower, and smart multi-tone jammers. As expected, in the presence of a partial band jammer, the proposed FH-ALE system performance gain compared to the traditional system is evident. For example, considering $P_{\text {link }}=95 \%$, the performance gain of exploiting FH-ALE system is almost 0.9 dB. Furthermore, in the case of follower jammer, utilizing proposed system results in an almost $2.5 \mathrm{~dB}$ performance gain for $P_{\text {link }}=95 \%$. Besides, proposed system performance gain is limited in smart multi-tone jammer case about $0.6 \mathrm{~dB}$ for $P_{\text {link }}=95 \%$, referring.

\subsection{The throughput comparison}

Since by reducing the probability of link establishment, the data transmission time is affected and reduced. So in different interference situations, the value of the proposed system throughput is compared and evaluated with the tradi- 


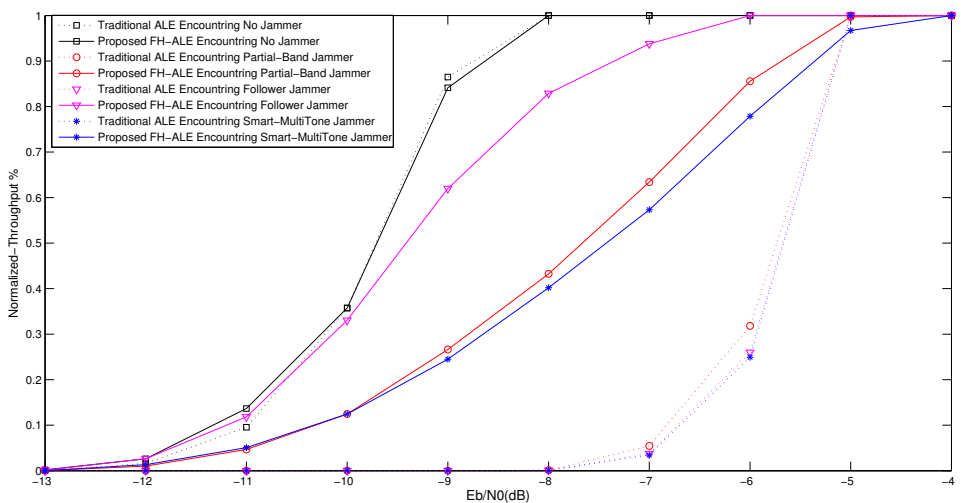

Fig. 7 The throughput comparison of the proposed FH-ALE approach and the traditional ALE system[6] encountering various jamming environment

tional system. The comparison between the proposed and traditional systems in different conditions, including the absence of jammer and the presence of other jammers, is shown in Fig. 7.

The results obtained for throughput show that in the absence of jamming and with the presence of a sweeper jammer, the proposed system will have almost the same performance as the traditional system. Also, in the presence of the partial band, follower, and smart multi-tone jammers, the throughput value in the proposed system increases compared to that of the traditional system, and the efficiency of the proposed system increases to about $1 \mathrm{~dB}$ to $4 \mathrm{~dB}$.

\section{Conclusion}

This paper represented an FH-based ALE algorithm that is robust in HF network and jamming environments. Three different jamming scenarios are considered in the simulations, including partial-band jammer, smart multitone jammer, and follower jammer. Due to the use of a channelizer in the Suggested system receiver, the time of the proposed FH-ALE approach is approximately identical to the traditional ALE system, and the throughput 
not only does not decrease but also increases in terms of interference. The simulation results represented the proposed FH-ALE approach's superiority over the traditional ALE criterion in jamming scenarios.

\section{References}

1. Interoperability and Performance Standards for Medium and High Frequency Radio Systems(1988), MIL-STD-188-141A, U.S. Dept. Defense.

2. Interoperability and Performance Standards for Medium and High Frequency Radio Systems(1999), MIL-STD-188-141B, U.S. Dept. Defense.

3. Bilal, A., Sun. G.(2017) "Automatic Link Establishment for HF Radios," ICSESS.

4. Interoperability and Performance Standards for Medium and High Frequency Radio Systems(2011), MIL-STD-188-141C, U.S. Dept. Defense.

5. Technical Standards for an Automatic Radio Control System (ARCS) for HF Communication Links(2000), STANAG 4538, North Atlantic Treaty Org.,ed. 1.

6. Yu. L., Chen. J., Ding. G., Qin. zh.(2016) "Fast automatic link establishment: A new metric and the value of spectrum prediction," WCSP.

7. Interoperability and Performance Standards for Medium and High Frequency Radio Systems(2017), MIL-STD-188-141D, U.S. Dept. Defense.

8. Technical standards for a Slow-hop HF EPM Communication Systems(1999), STANAG 4444, North Atlantic Treaty Org.

9. Bejar-Haro. B., Zazo-Bello. S., Perez-Alvarez. I., Quintana. A., and Camas. J. M.(2009) "STANAG 4444: Improved soft-decision detector for the spread spectrum mode," IRST.

10. Wenlong. Y., Yunpeng. C., and Liang. S.(2011) "Adaptive Frequency-Hopping in HF Communications," IEEE Int. Conf., pp. 427-430.

11. Wang. Y, Niu. Y., Chen. J., Fang. F., Han. C.(2019) "Q-Learning Based Adaptive Frequency Hopping Strategy Under Probabilistic Jamming," 11th International Conference on Wireless Communication and Signal Processing.

12. Ye. P., Wang. Y., Li. J., Xiao. L.(2020) "Fast Reinforcement Learning for Anti-Jamming Communication," Electrical Engineering and Systems Science.

13. Kadhim. A.A., Sadkhan. S.B.(2020) "Cognitive Radio Performance Enhancement Based on Frequency Hopping System," IT-ELA.

14. Interoperability and Performance Standards for Advanced Adaptive HF Radio, MILSTD-187-721D(1999), U.S. Dept. Defense.

15. Sprague. R.A., Babu. P.(2008) "A new propagation prediction tool for earth-space geometries for the advanced refractive effects prediction system (AREPS)," MILCOM .

16. Stocker. A.J.(2012) "Fast and accurate calculation of multipath spread from VOACAP predictions," Radio Science., Volume: 47, Issue: 04. 
17. Khoder. K., Fleury. R., Pagani. P.(2014) "Monitoring of ionosphere propagation conditions using opportunistic HF signals," EuCAP.

18. Lifeng. W., Zhigang. Zh.(2019) "The Calculation of HF Ground Wave Propagation over Complex Terrain Based on FEKO/PE Hybrid Algorithm" ICAICA.

19. Hassan. Sh., Bokhari. M.U.(2019) "Design of Pseudo Random Number Generator using Linear Feedback Shift Register," IJEAT, vol. 9, Issue. 2, pp. 1956-1965.

20. Liu. X., Xu. Y., Cheng. Y., Li. Y., Zhao. H., Zhang. X.(2018) "A heterogeneous information fusion deep reinforcement learning for intelligent frequency selection of $\mathrm{HF}$ communication," China Commun., vol. 15, no. 9, pp. 73-84.

21. Qin. Zh., Wang. J., Chen. J., Ding. G., Yao. Y.D., Ji. X., Chen. X.(2018) "Link Quality Analysis Based Channel Selection in High-Frequency Asynchronous Automatic Link Establishment: A Matrix Completion Approach," IEEE Syst. J., vol. 12, no. 2.

22. Chen. X., Harris. F., Venosa. E.(2012) "Polyphase channelizer for fully digital frequency hopping systems," in Analog Integr Circ Sig Process, pp. 517-530.

23. Jang. Y., Kim. Gh., Park. B., Lim. H.(2021) "Generalized Polyphase Digital Channelizer," in IEEE Transactions on Circuits and Systems.

24. Johnson. E.E.(2015) "Staring link establishment for high-frequency radio," in Military Communications Conference, MILCOM 2015, pp. 1433-1438.

25. Johnson. E.E.(2016), "WIDEBAND ALE-THE NEXT GENERATION OF HF," in Nordic HF Conference, HF, vol. 16.

26. Ivanov. D.V., Ivanov. V.A., Ovchinnikov. V.V.,Ryabova. M.I.(2019) "Method of Training Mode of Adaptive System for Frequency Dispersion Correction in Wideband Ionospheric HF Communication Channels," WECONF.

27. Harris. F., Chen. X., Venosa. E.(2015) "Efficient Implementation of Multicarrier Frequency Hopping reciever via Polyphase Channelizer," in IEEE Military Communications Conference.

28. Ghandour. A., Mansour. A., Alasadi. H., Ghandour. W.(2020) "Design and Implementation of Polyphase Fast Fourier Transform Channelizer," in IWCMC.

29. Xiao. L., Jiang. D., Xu. D., Zhu. H., Zhang. Y.(2018) "Two Dimensional Antijamming Mobile Communication Based on Reinforcement Learning," IEEE Transaction on Vehicular TeChnology, vol. 67. 